Original Research Paper

\title{
Automatic Recognition System of Vehicle Plate for Recording Incoming Vehicles to the University Campus and the Outgoing
}

\author{
${ }^{1}$ A.A. El-Harby, ${ }^{2}$ G.M. Behery and ${ }^{3}$ A.A. Al-Dhawi \\ ${ }^{1,2}$ Department of Computer Science, Community College in Dammam, University of Dammam, KSA \\ ${ }^{2}$ Department of Mathematics, Faculty of Science, University of Dammam, KSA
}

\author{
Article history \\ Received: 22-04-2016 \\ Revised: 25-12-2016 \\ Accepted: 29-03-2017 \\ Corresponding Author: \\ A.A. El-Harby \\ Department of Computer \\ Science, Community College in \\ Dammam, University of \\ Dammam, KSA \\ Email: aelharby@uod.edu.sa
}

\begin{abstract}
Automatic recognition of vehicle plate characters became a very important in our daily life because of the unlimited increase of vehicles that enter or leave a supervised area. This paper describes an automatic intelligent system that captures the images of vehicles and has the ability to recognize the plates of vehicles entering the university campus or leaving. A camera is built on the university gate for taking images of incoming or outgoing vehicles. A rear image of a vehicle is captured and processed. The system applies an intelligent filtering of the input image based on a set of filters removing unnecessary image elements preserving the position and shape of characters of the vehicle plate, histogram manipulation and edge detection techniques for plate localization and characters segmentation. Adaptive Neuro Fuzzy Inference System (ANFIS) is chosen as a classifier for recognizing the characters in the vehicle plates. This system can extracts the letters and numbers from vehicles plates. So, the extracted vehicle characters can be used to record the incoming vehicles to the university campus and the outgoing. The recognized vehicle characters are saved in the university database.
\end{abstract}

Keywords: Vehicle Plate Recognition, ANFIS System, Feature Extraction

\section{Introduction}

License Plate Recognition (LPR) was detected using some applications. These applications were used for identifying vehicles in a restricted area and for detecting stolen vehicles (Kranthi et al., 2011; Chenhong and Zhaoyang, 2008; Hongliang and Changping, 2004; Sunghoon et al., 2002; Sang et al., 1996). Recognition of license plates using traffic video databases was carried out on global edge features with various parameters based on blob detection algorithm. The LPR results due to a faster and more precise identification (Lihong et al., 2013; Jianbin et al., 2009). Well-organized system has the ability to translate Arabic text found in images into English. This system can detect images, character recognition and machine translation. The experimental results show improvements in accuracy for word recognition (Chang et al., 2009). Efficient algorithms can detect license plate location from car images. Their results show that the proposed methods are more effective (Ioannis et al., 2010; Danian et al., 2005; Nicolás et al., 2012).
This method was applied on database, containing car images taken from different distances and viewing angels. The method was given a precise evaluation with other related work (Vahid and Alireza, 2009). The license plate based strategy can identify means of integrated horizontal and vertical projections that are scanned using a search window. This system was carried out on license plate images in effective way. Experiments show that a recognition rate was $95.7 \%$ (Yo-Ping et al., 2009). Two algorithms were used to map a car plate image into an invariant vector magnitude based on Gaussian low pass filter. They are robust to the disturbances for instances non-uniform brightness distribution on the different positions of a license plate images using various colors. The algorithms can precisely detect car plate images (Ching-Liang, 2011; Sedighi and Vafadust, 2011). A license plate image is identified without the depending on the location of the license plate. Then, this system can determine the accurate location of a license plate. The mean shift was used to make filtering and segmentation of a color vehicle image providing candidate regions. These 
regions were then classified to choose a candidate region contains a license plate (Wenjing et al., 2007).

An automatic method for vehicle identification was applied using a plate clip, character segmentation and recognition. The recognition process was used an adaptive iterative threshold algorithm. This method was proposed based on an invariant to use a suitable character size and thickness for identifying vehicle license plate (Vladimir et al., 2006). The proposed system was tested on big database containing 1334 vehicle images having different backgrounds. The license plates were correctly segmented with performance equal to $96.5 \%$. The architecture of this system was designed with two layers. This system was trained to classify alphanumeric characters from car plates. The system can identify characters from license plates images without the need to specify the size and inclination of the license plate or the color of the vehicle (Christos et al., 2006).

For identifying the vehicles of a garage entrance, a camera was fixed on the door; so, cars that entered can be detected and matched to the database of authenticated cars. Then, LPR was applied based on the character detection and recognition. After that the license plate number was matched with the database of authenticated plates. If the car was allowed access, the door will be opened in automatic way (Rob and Peter, 2011). This system was proposed to detect car number plate for Malaysian. It was used matching technique to approximate the location of the number plate region. It was found that the number plate region can be determined more accurately (Mohd and Suandi, 2010).

The problem of our concern is: Given a vehicle license plate image and apply preprocessing techniques to enhance this image. The image is converted to black and white. Then, isolate the license plate and apply histogram followed by edge detection techniques. After that, separate the areas of Arabic and English for letters and numbers. Finally apply the AI system to recognize the license plate information. The proposed algorithm is able to find a better solution. Extensive simulations have been conducted and the results have evaluated of the proposed algorithm.

The paper is organized as follows. In section 2, we describe the feature extraction process in general. The section 3 is used to present the proposed system in more details. Section 4 shows the image segmentation and feature extraction model and the designed functions for the proposed system. Section $5 \%$ the structure, the training and the test processes of ANFIS algorithm. Section 6 includes the results, discussion and comparing with others. Section 7 concludes the system and provides future work.

\section{Feature Extraction}

A complete image cannot be used or processed every time as the required memory may be large. Feature extraction process can reduce the processing speed.
Hence only prominent features are extracted from each image and an image feature database is formed. Also pixel by pixel comparison with the query image may consume most of the time of the processor and a shift by one pixel value may lead to complete mismatch and pseudo results (Patrick, 2011).

Feature extraction involves detecting and isolating various desired features of patterns. It is the operation of extracting features for identifying or interpreting meaningful information from the data. This is especially relevant in the case of image data where feature extraction involves automatic recognition of various features (Murty and Devi, 1988). Eigen feature extraction is proposed (Latha and Devarajan, 2014). Coarse grid, Directional element, Invariant moment and Permeability Number features are presented (Kranthi et al., 2011; Miguel et al., 2010). Conventional, Gradient Directional Gradient, Directional Gradient Features Extraction are presented (Singh et al., 2014). The Cross-Corner Feature Extraction is presented by (Manju and Yogesh, 2011). Shadow and 64 dimensional features are purposed by (Deepali and Manasi, 2015). Finally the author will apply some of features extraction to collect the training and test data.

Features are characteristic properties of the objects whose values should be similar for objects in a particular class and different from the values for objects in another class. Features may be continuous or categorical. Examples of continuous variables would be length, area and texture. Categorical features are either ordinal or nominal. The choice of appropriate features depends on the particular image and the application at hand. However, they should be robust, discriminating, reliable and independent (Anke and Volker, 2014).

A good feature should remain unchanged if variations take place within a class and it should reveal important differences when discriminating between patterns of different classes. In other words, patterns are described with as little loss as possible of pertinent information. There are four known categories in the literature for extracting features:

1. Non-transformed structural characteristics: moments, power, amplitude information, energy, etc. Let $I(x, y)$ is a continuous image function. The geometric moment equation of order $p+q$ is:

$m_{p q}=\int_{-\infty}^{\infty} \int_{-\infty}^{\infty} x^{p} y^{q} \mathrm{I}(x, y) d x d y$

Similarly, let $I(x, y)$ is a digital image with $i=0,1, \ldots, N_{x}-1, \quad j=0,1, \ldots, N_{y}-1 \quad$ the corresponding moment equation of order $p+q$ as in Equation 2:

$m_{p q}=\sum_{i} \sum_{j} I(x, y) i^{p} j^{q}$ 
2. Transformed structural characteristics: Frequency and amplitude spectra, subspace transformation methods, etc

3. Structural descriptions: Formal languages and their grammars, parsing techniques and string matching techniques

4. Graph descriptors: Attributed graphs, relational graphs and semantic networks (Anke and Volker, 2014)

\section{The Proposed System}

The process of identifying data plate for cars in Saudi Arabia mechanically by cameras installed in specific places at traffic lights or other places so necessary to help maintain discipline street traffic in all cities and villages of the Kingdom. It is also very necessary in a particular University because it provides a database of license plate numbers and time of entry to the campus, as well as license plate numbers and the time they leave campus.

Automatic License Plate Recognition (ALPR) is one of the most important aspects of applying computer techniques towards building intelligent system. A camera is built on the university gate for taking images of incoming or outgoing vehicles. ALPR is the extraction of vehicle license plate information from an image or a sequence of images used to identify a vehicle by its license plate. The extracted information can be used with or without a database. The system processes are firstly captured a rear image of a vehicle. Then, an intelligent filtering is applied for removing unnecessary image elements preserving the position and shape of characters of the vehicle plate. The image is also changed from color into black and white. License plate location is an important stage in vehicle license plate recognition for automated system (https://goo.gl/vLRPn6).

The main stage is the isolation of the license plate, from the digital image of the car obtained by a digital camera under different circumstances such as illumination, slop, distance and angle. Then, histogram manipulation and edge detection techniques are used to identify the location of vehicle plate; Fig. 1a. After that, the segmentation process is applied to separate the areas of Arabic letters, English letters, Arabic numbers and English numbers; Fig. 1b. ANFIS system is applied as a classifier for recognizing each character in the vehicle plate. Finally, the recognized vehicle characters are saved in the database.

The detailed steps of the proposed algorithm are given as follow:

1. Capture the image using Matlab preview function. img $=$ preview (obj, target-Image).

2. Store the captured image into an image file for further processing.

3. Resize the capture image into $140 \times 260 \times 3$.

4. Transfer the image to gray level image.
5. Filter the noise level present in the image.

6. Transfer the gray image to black and white image

7. Enhance the $B W$ image.

8. Segmentation process as follows (Fig. 1):

a-Divide the image into two parts of characters and label.

$b$ - Clip the plate area in such a way that separates numbers (Indian and Arabic) of plate area extracted.

c-Clip the plate area in such a way that separates alphabetic (English and Arabic) of plate area extracted.

$d$-Separate each character from the plate.

9. Resize all characters into $64 \times 64$.

10. Extract the features

11. Build the training and test data.

12. Design the ANFIS system (Fig. 6).

13. Train the system on the training data.

14. Test the system on test data.

15. Apply the system on the separated characters from the plate.

16. Get the results.

17. End.

\section{Preparing Data}

The pattern data is used for training and testing the system. This data contains 296 images representing the Arabic letters, Arabic numbers, English letters and Indian numbers. These images are constructed using different font sizes. The images are divided into two parts; they are 75 and $25 \%$. The two data parts are used for training and test respectively. Features extraction processes are applied as preprocessing on each image; see the next subsection for more details. The image size must be modified to $2^{p} \times 2^{q} \times 3$, where $p$ and $q$ are integer numbers.

\section{Image Segmentation and Feature Extraction Model}

Each image is firstly divided into 48 partitions. A feature is extracted from each partition. This division is constructed through four stages. The first stage contains five partitions; the whole image, two halves vertically and two halves horizontally; Equation 3 and Fig. 2:

$$
\left.\begin{array}{l}
S 2=\mathrm{A}\left(0: n, 0: \frac{m}{2}\right) \\
S 3=\mathrm{A}\left(0: n, \frac{m}{2}+1: m\right) \\
S 4=\mathrm{A}\left(0: \frac{n}{2}, 0: m\right) \\
S 5=\mathrm{A}\left(\frac{n}{2}+1: n, 0: m\right)
\end{array}\right\}
$$

where, $S 2, S 3, S 4$ and $S 5$ represent the four partitions, $n$ and $m$ are the number of rows and columns respectively. $S 1 \_5$ represents the partitions 1 up to 5 . 


\begin{tabular}{|c|c|c|}
\hline Endian Numbers & Arabic Alphabet & \\
\hline Arabic Numbers & English Alphabet & \\
\hline
\end{tabular}

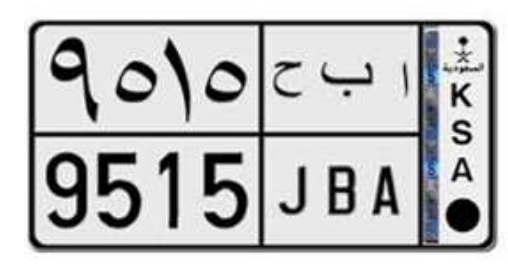

(a)

\begin{tabular}{|l|l|}
\hline Endian Numbers & Arabic Alphabet \\
\hline Arabic Numbers & English Alphabet \\
\hline
\end{tabular}$\quad$\begin{tabular}{|}
\hline \\
\hline
\end{tabular}

\begin{tabular}{|l|}
\hline Endian Numbers \\
\hline Arabic Numbers \\
\hline
\end{tabular}$\quad$\begin{tabular}{|l|}
\hline Arabic Alphabet \\
\hline English Alphabet \\
\hline
\end{tabular}

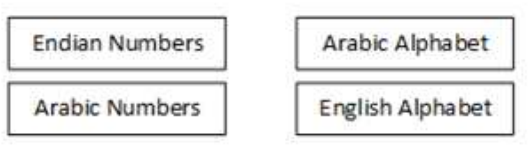

\begin{tabular}{|c|c|c|c|c|c|c|}
\hline 9 & 0 & 1 & 0 & $\tau$ & ب & 1 \\
\hline 9 & 5 & 1 & 5 & $\mathrm{~J}$ & B & A \\
\hline
\end{tabular}

(b)

Fig. 1. (a) Original Image plate (b) Segmented areas
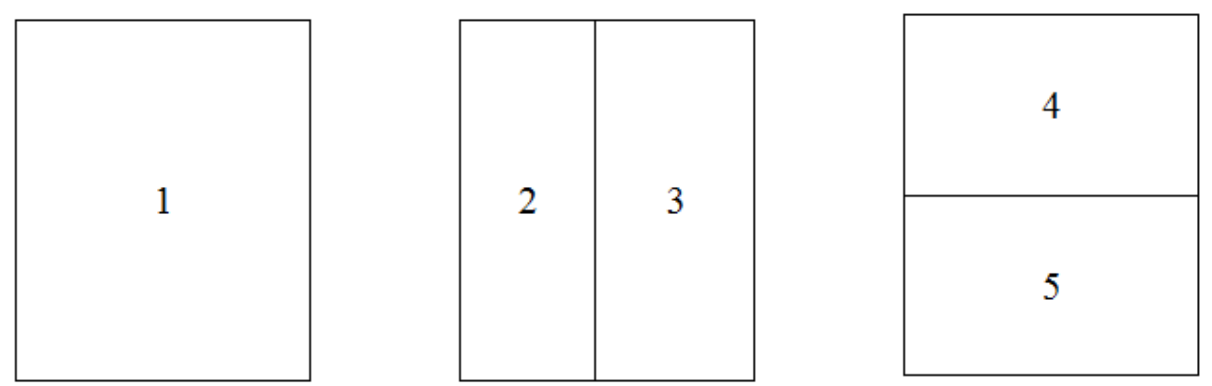

Fig. 2. Partitions 1-5 of the image

\begin{tabular}{|l|l|}
\hline 6 & 7 \\
\hline 8 & 9 \\
\hline
\end{tabular}

\begin{tabular}{|c|c|}
\hline 10 & 11 \\
\hline 12 & 13 \\
\hline 14 & 15 \\
\hline 16 & 17 \\
\hline
\end{tabular}

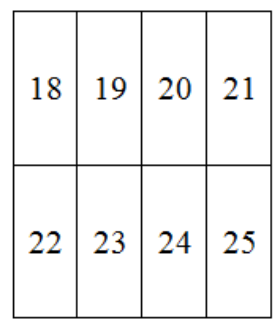

\begin{tabular}{|l|l|l|l|}
\hline 26 & 27 & 28 & 29 \\
\hline 30 & 31 & 32 & 33 \\
\hline 34 & 35 & 36 & 37 \\
\hline 38 & 39 & 40 & 41 \\
\hline
\end{tabular}

Fig. 3. Partitions 6-41 of the image
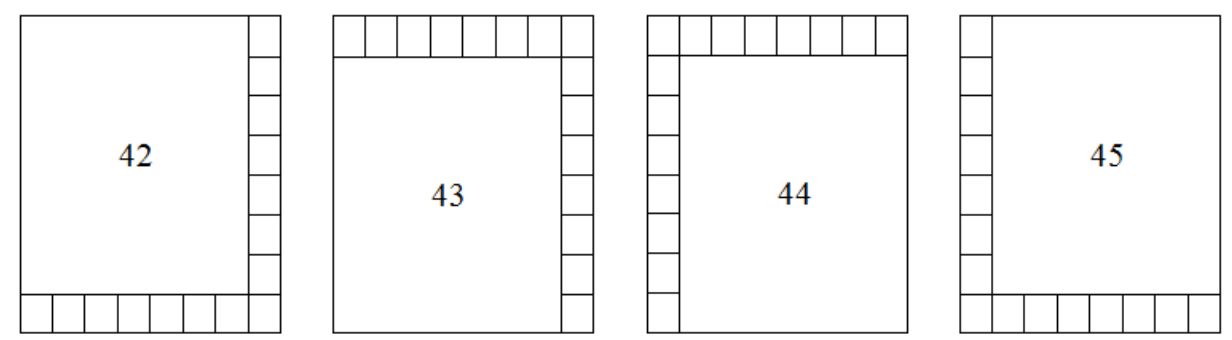

Fig. 4. Partitions $42-45$ of the image 

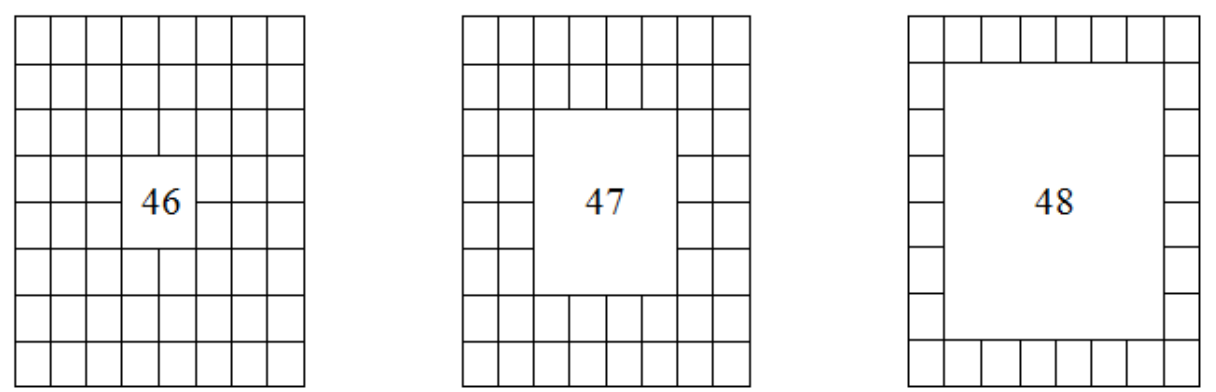

Fig. 5. Partitions $46-48$ of the image

The second stage contains 36 partitions started from 6 up to 41. Firstly, the whole image is divided into 4 partitions. Then, the whole image is divided into 8 partitions vertically and horizontally. Finally the image is divided into 16 partitions with the corresponding marks as shown by Equation 4 and Fig. 3:

$S 6 \_41=A\left(\begin{array}{l}i+\frac{i * n}{C}: i+\frac{i^{*} n}{C}+\frac{n}{C}, \\ j+\frac{j^{*} m}{R}: j+\frac{j^{*} m}{R}+\frac{m}{R}\end{array}\right)$

where, S6_41 represents the partitions 6 up to 41 considering the row number $i$ and the column number $j$. The horizontal and vertical numbers of blocks are $C$ and $\mathrm{R}$ respectively.

The third stage includes four cases divided diagonally, the first two starting from left to right and the last two from right to left; Equation 5 and Fig. 4:

$$
\begin{aligned}
& \left.S 42=A\left(0: n-\frac{n}{8}, \quad 0: m-\frac{m}{8}\right)\right) \\
& S 43=A\left(\frac{n}{8}+1: n, \quad 0: m-\frac{m}{8}\right) \\
& S 44=A\left(\frac{n}{8}+1: n, \quad \frac{m}{8}+1: m\right) \\
& \left.S 45=A\left(0: n-\frac{n}{8}, \frac{m}{8}+1: m\right)\right)
\end{aligned}
$$

where, $S 42-S 45$ represent the partitions 42 up to 45 .

The last stage contains three partitions. Partition 46 is made by taking the innermost 4 cells. Partition 47 is done by taking 16 innermost cells. Partition 48 is made by taking 32 innermost cells; see Equation 6 and Fig. 5 . These partitions are used to extract the features that are built the required database:

$$
S 46 \_48=A\left(\begin{array}{l}
\frac{n^{*} B}{8}+1: n-\frac{n^{*} B}{8}, \\
\frac{m^{*} B}{8}+1: m-\frac{m^{*} B}{8}
\end{array}\right)
$$

where, $B=3,2$ and 1 for the partitions 46-48.

That is means, the input data to the system are 48 features was their pulling out from the base of the verified data consisting of 10 preparing Arabic and Hindi numbers 10 and 26 English letters and 28 Arabic letters in four different sizes, 18, 22, 26, 30 and one fixed fonts because printed plates all fonts one. This means that the database consists of 296 image size $64 \times 64$.

\section{ANFIS Algorithm}

Neuro-fuzzy refers to the combination of fuzzy set theory and neural networks (Zounemat-Kermani et al., 2009). Jyh-Shing Roger Jang proposed a novel architecture called ANFIS (Jang, 1993), which conserve as a basis for constructing a set of fuzzy if-then rules with appropriate membership functions to generate the stipulated input-output pairs. These techniques provide the fuzzy modeling procedure to learn information about a data set, in order to compute the membership function parameters that give best allow of the associated fuzzy inference system to track the given input-output data. This learning method works similarly to that of neural networks (Dlala and Arkkio, 2006). ANFIS implements a Tokagi Sugeno FIS having a six layered architectures as shown in Fig. 6. This figure is drown based on (https://goo.gl/rc5jyH).

Layer 1: The first layer of this architecture consists of a membership degree of input variables (features extraction) to corresponding terms: $\mu_{i j}=\mu_{i}\left(x_{j}, P_{i}\right)$, where $j=1,2, \ldots, N-N$ is number of features, $x_{j}$ is the calculated $\mathrm{j}^{\text {th }}$ feature, $i=1,2, \ldots, L, L$ is the number of given terms, $P_{i}$ is the vector of membership function parameters of $i^{\text {th }}$ term. For example: For membership functions, generalized bell membership function (Equation 7), a Gaussian membership function (Equation 8), or any membership functions:

$\mu_{i j}(\mathrm{x})=\frac{1}{1+\left|x-\frac{c_{i}}{a_{j}}\right|^{2 b_{j}}}, j=1,2, \cdots N$ 


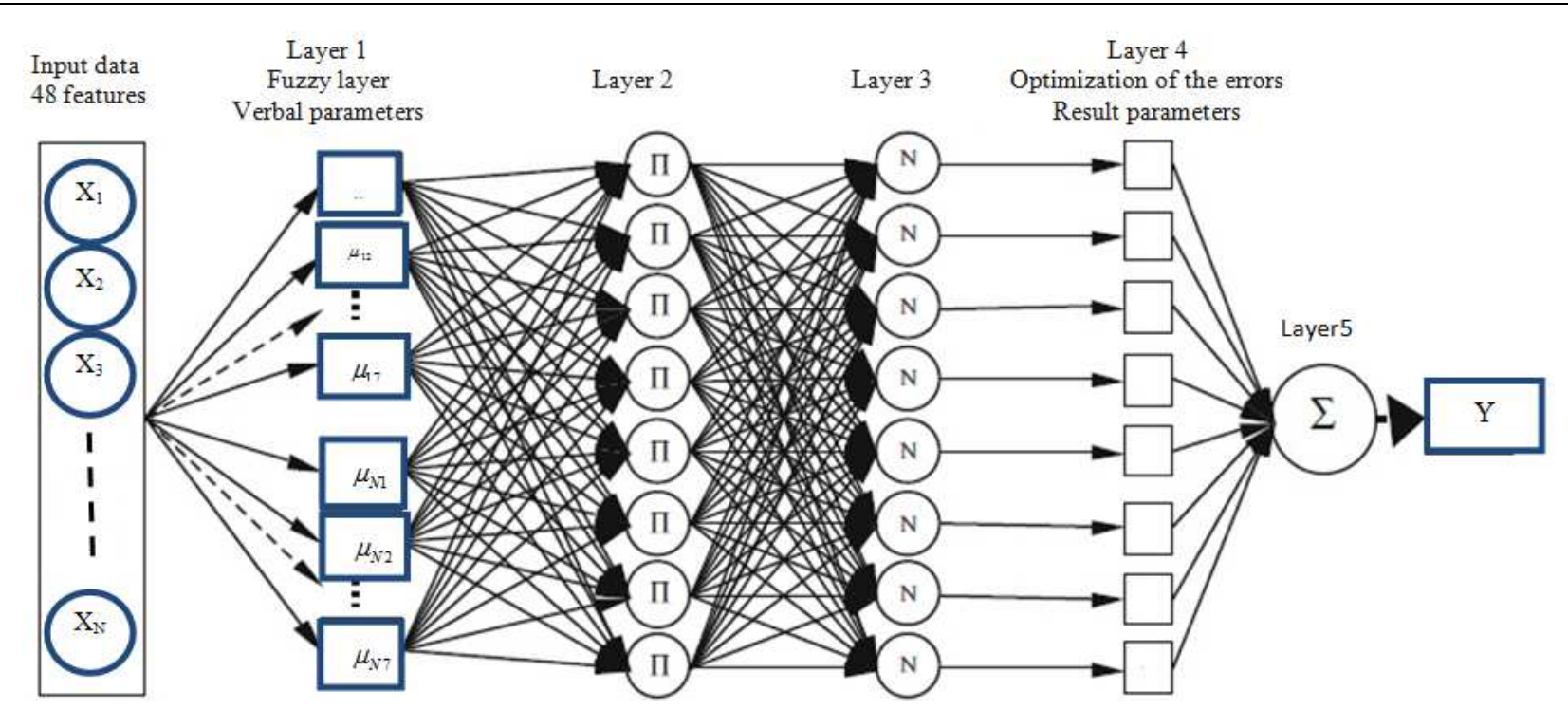

Fig. 6. ANFIS architecture based on Takagi-Sugeno model

$$
\mu_{i j}(x)=\exp \left[-\left(\frac{x-c_{j}}{a_{j}}\right)^{2}\right], \quad j=1,2, \cdots N
$$

where, $\left\{a_{i}, b_{i}, c_{i}\right\}$ is a set of the premise parameters.

Layer 2: Every node in this layer is a fixed node, marked by a circle, labeled $\Pi$ and is calculated by the fuzzy "AND" connective of "PRODUCT" of the incoming signals, i.e., П-norm operation (Equation 9):

$$
w_{i}=\mu_{A j}\left(x_{k}\right) \times \mu_{B m}\left(x_{z}\right)
$$

Layer 3: The nodes of third layer calculates the ratio of given rule to the sum of all rules' values as in Equation 10:

$$
\bar{w}_{i}=\frac{w_{i}}{\sum_{i=1}^{N} w_{i}}
$$

Layer 4: Each node in the fourth layer is connected with a node of the third layer and inputs of the network. The node of the fourth layer counts a contribution of one fuzzy rule to a network exit as in the following Equation 11:

$$
y_{i}=\bar{w}_{i}\left(b_{0 i}+b_{1 i} x_{1}+b_{2 i} x_{2}+\cdots+b_{N i} x_{N}\right)
$$

where, $\quad\left\{b_{k i}, \quad k=1, \cdots, N, \quad i=1, \cdots L\right\} \quad$ are the set of parameters.

Layer 5: The single node of the last layer computes the overall output using center average defuzzification method.

In order to evaluate the proposed ANFIS algorithm, a comparison is made in the last subsection. The obtained results of the ANFIS system are presented in the following subsections, respectively. All programs are written using the MATLAB software.

The proposed system is designed to find the best ANFIS that has the ability to have the best recognition of vehicle license plates by changing the type and number of membership functions and the number of epochs. Thus, many attempts are made to find the best ANFIS that uses a low number of epochs and membership functions. The experiments are trained using the proposed numbers of membership functions for 100 epochs and continue up to 2000 epochs. The ANFIS system reached the optimal solution using 1500 epochs and seven membership function with the 'gbellmf' membership. The proposed system is trained. After the training, it is found that the results using two up to six membership functions are not enough. The results at seven membership functions are good, having the best result at 1500 epochs. The system was trained and tested using the two main database sets respectively and the overall average performance was $98.6 \%$.

\section{Results and Discussion}

The proposed system using image processing and the best trained ANFIS is applied on 150 plates, part of them are taken from (https://goo.gl/vLRPn6) and the other by camera from different distances and angles. The ANFIS algorithm reached the optimal performance using the 7 membership functions at 1500 epochs. Figure 7 shows the results for recognizing one plate, the plate and ANFIS result are given in the Fig. $a$ and $b$ respectively. The results showed that, the average of performance for Arabic digits, Indian digits, Arabic letters and English letters are 97.4, 94.6, 96.2 and 96.7\% respectively. 


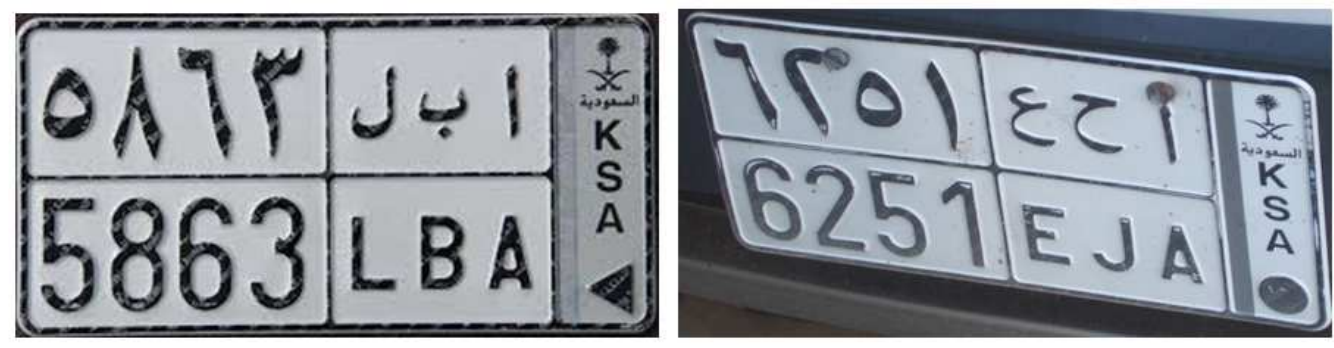

(a)

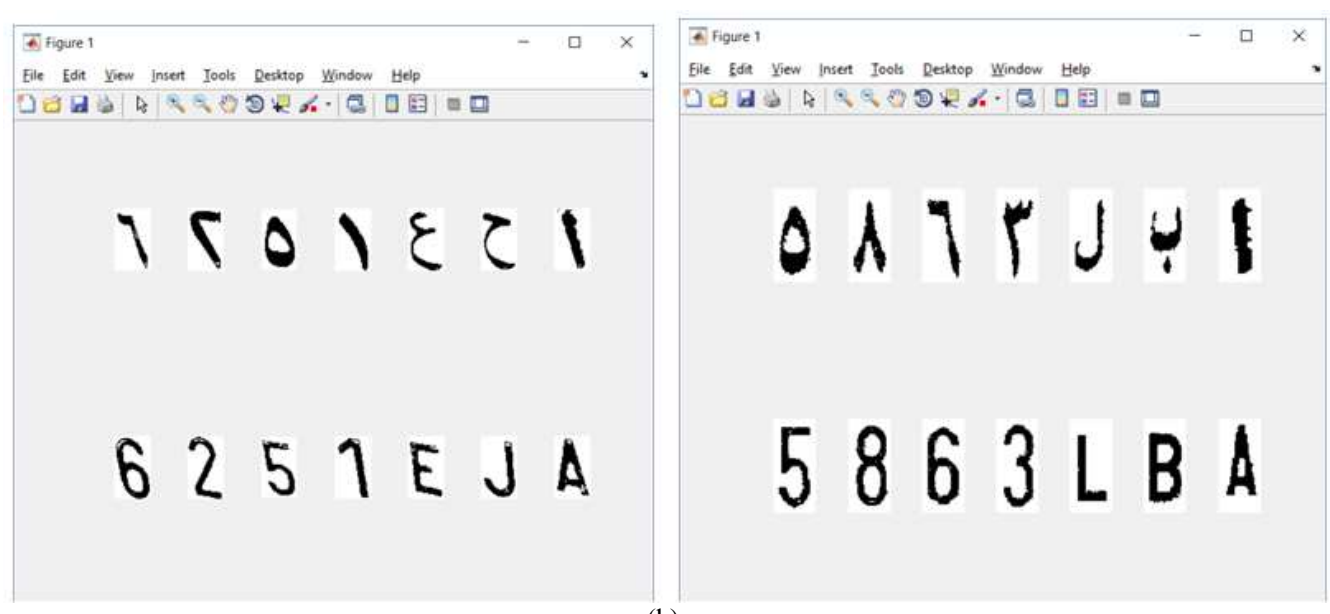

(b)

Fig. 7. (a) Vehicle plates (b) ANFIS results

Table 1. The obtained performances

\begin{tabular}{llllll}
\hline Average & Arabic digit & Indian digit & Arabic letter & English letter & Overall average \\
\hline Success rate & $\mathbf{\% 9 7 . 4}$ & $\mathbf{\% 9 4 . 6}$ & $\mathbf{\% 9 6 . 2}$ & $\mathbf{\% 9 6 . 7}$ & $\mathbf{\% 9 6 . 2 3}$ \\
\hline
\end{tabular}

Table 2. Comparison with other researches

\begin{tabular}{|c|c|c|c|c|c|c|c|}
\hline & $\begin{array}{l}\text { Syed et al. } \\
(2013)\end{array}$ & $\begin{array}{l}\text { Sandeep et al. } \\
(2013)\end{array}$ & $\begin{array}{l}\text { Tahir et al. } \\
(2010)\end{array}$ & $\begin{array}{l}\text { Deriche and Zidouri } \\
(2008)\end{array}$ & $\begin{array}{l}\text { Al-Audah et al. } \\
(2012)\end{array}$ & $\begin{array}{l}\text { Yusuf et al. } \\
(2014)\end{array}$ & $\begin{array}{l}\text { Proposed } \\
\text { System }\end{array}$ \\
\hline Arabic digits & $\mathrm{N} / \mathrm{A}$ & $\mathrm{N} / \mathrm{A}$ & N/A & $\mathrm{N} / \mathrm{A}$ & N/A & $\mathrm{N} / \mathrm{A}$ & \%97.4 \\
\hline Indian digits & N/A & N/A & N/A & N/A & N/A & $\% 91.6$ & $\% 94.6$ \\
\hline Arabic letters & N/A & N/A & N/A & N/A & N/A & $\% 96.55$ & $\% 96.2$ \\
\hline English letters & $\% 98$ & N/A & N/A & N/A & N/A & N/A & $\% 96.7$ \\
\hline Overall average & N/A & $\% 83.84$ & $\% 92.1$ & $\% 93$ & $\% 94$ & $\% 94.08$ & $\% 96.23$ \\
\hline
\end{tabular}

It is found that the results obtained indicate that ANFIS is more effective and feasible specially for extracting Arabic letters, English letters and Arabic digits. It is also noticed that the performance with extracting Indian digits is slightly low comparing with the obtained performances of Arabic letters, English letters and Arabic digits; see Table 1. The overall average performance is $96.23 \%$ for recognizing the above mentioned four characters' types.

In order to evaluate the proposed system, it is compared with other researches. The performances of them are calculated as overall average. Moreover, this comparison shows that the overall average performance of the proposed system is more efficient from the others especially for extracting Arabic letters, English letters and Arabic digits as shown in Table 2.

\section{Conclusion}

This paper highlighted the importance of identifying information on the metal plate in Saudi Arabia automatically using the computer, depending on image processing and artificial intelligence. The proposed system developed KSA license plate recognition system using the image processing and ANFIS system. The pattern data contains images of the Arabic letters, Arabic numbers, English letters and Indian numbers. Hence, each image is divided into 48 partitions to extract a feature from each partition. These features are detailed 
mathematically in section 3.1. The ANFIS algorithm is trained and tested to choose the best one as a classifier.

The proposed system is applied on many KSA license plates, it is found that the obtained results are more efficient for extracting the Arabic letters, Indian numbers, English letters and Arabic numbers. The obtained performances for the above four characters types are shown in Table 1 and theoverall average performance is $96.23 \%$. The results are compared with other researches. The performances of them are calculated as overall average. Moreover, this comparison shows that theoverall average performance of the proposed system is the best.

It is also found that the proposed system is not treated well with the Arabic letter 'I' because its width is very thin and we will try to develop this system in the future to solve this problem.

\section{Acknowledgement}

We would like to thank the Deanship of Scientific Research at the University of Dammam, KSA for funding this research.

\section{Funding Information}

This research is funded by the Deanship of Scientific Research at the University of Dammam, KSA.

\section{Author's Contributions}

All authors completed the paper together as well as read and approved the final manuscript.

\section{Ethics}

It is declared that there are no ethical issues that may arise in the present study.

\section{References}

Al-Audah, Y.K., A.K. Al-Juraifani and M.A. Deriche, 2012. A real-time license plate recognition system for Saudi Arabia using lab VIEW. Proceedings of the 3rd International Conference on Image Processing Theory, Tools and Applications, Oct. 15-18, IEEE Xplore Press, Saudi Arabia.

DOI: 10.1109/IPTA.2012.6469576

Anke, M.B. and S. Volker, 2014. Pattern Recognition and Signal Analysis in Medical Imaging. 2nd Edn., Elsevier Inc., pp: 25.

Chang, Y.C., C. Datong, Z. Ying and Y. Jie, 2009. An image-based automatic Arabic translation system. Patt. Recog., 42: 2127-2134. DOI: $10.1016 /$ j.patcog.2008.10.031

Chenhong, L. and L. Zhaoyang, 2008. Local feature extraction for iris recognition with automatic scale selection. Image Vis. Comput., 26: 935-940. DOI: $10.1016 /$ j.imavis.2007.10.011
Ching-Liang, S., 2011. Car plate recognition by whole 2-D image. Expert Syst. Applic., 38: 7195-7200. DOI: $10.1016 /$ j.eswa.2010.12.039

Christos, N.E.A., I.E. Anagnostopoulos, V. Loumos and E. Kayafas, 2006. A license plate-recognition algorithm for intelligent transportation system applications. IEEE Trans. Intell. Transport. Syst., 7: 377-392. DOI: 10.1109/TITS.2006.880641

Danian, Z., Y. Zhao and J. Wang, 2005. An efficient method of license plate location. Patt. Recog. Lett., 26: 2431-2438. DOI: 10.1016/j.patrec.2005.04.014

Deepali, R.B. and M.P. Manasi, 2015. Recognition of off-line handwritten devanagari characters using combinational feature extraction. Int. J. Comput. Applic.

Deriche, M. and A. Zidouri, 2008. Recognition of Arabic license plates using NN. Proceedings of the 1st Workshops on Image Processing Theory, Tools and Applications, Nov. 23-26, IEEE Xplore Press, Saudi Arabia. DOI: 10.1109/IPTA.2008.4743757

Dlala, E. and A. Arkkio, 2006. A neuro-fuzzy-based Preisach approach on hysteresis modeling. Phys. B: Condensed Matter, 372: 49-52. DOI: 10.1016/j.physb.2005.10.017

Hongliang, B. and L. Changping, 2004. A hybrid license plate extraction method based on edge statistics and morphology. Proceedings of the 17th International Conference on Pattern Recognition, (ICPR' 04), China, pp: 831-834.

Ioannis, G., C.N. Anagnostopoulos, V. Loumos and E. Kayafas, 2010. Operator context scanning to support high segmentation rates for real time license plate recognition. Patt. Recog.., 43: 3866-3878. DOI: $10.1016 /$ j.patcog.2010.06.008

Jang, J.S.R., 1993. ANFIS: Adaptive-network-based fuzzy inference systems. IEEE Trans. Syst. Man Cybernet., 23: 665-685. DOI: 10.1109/21.256541

Jianbin, J., Q. Ye and Q. Huang, 2009. A configurable method for multi-style license plate recognition. Patt. Recog., 42: 358-369. DOI: $10.1016 /$ j.patcog.2008.08.016

Kranthi, S., K. Pranathi and A. Srisaila, 2011. Automatic number plate recognition. Int. J. Adv. Tech., 2: 408-422.

Latha, J. and N. Devarajan, 2014. Feature extraction of handwritten numeric characters and recognition using an artificial neural network: A new approach. J. Vibrat. Control, 20: 1869-1876. DOI: $10.1177 / 1077546313481003$

Lihong, Z., X. He, B. Samali and L.T. Yangc, 2013. An algorithm for accuracy enhancement of license plate recognition. J. Comput. Syst. Sci., 79: 245-255. DOI: $10.1016 /$ j.jcss.2012.05.006

Manju, R. and K.M. Yogesh, 2011. An efficient feature extraction method for handwritten character recognition. Proceedings of the 2nd International Conference SEMCCO, Dec. 19-21, Visakhapatnam, Andhra Pradesh, India, pp: 302-309. DOI: 10.1007/978-3-642-27242-4_35 
Miguel, A.D., S. Vega-Pons and E. Garea, 2010. Experimental comparison of orthogonal moments as feature extraction methods for character recognition. Proceedings of the 15th Iberoamerican Congress on Pattern Recognition CIARP, Nov. 8-11, Sao Paulo, Brazil, pp: 394-401. DOI: $10.1007 / 978-3-642-16687-753$

Mohd, F.Z. and S.A. Suandi, 2010. Malaysian car number plate detection system based on template matching and colour information. Int. J. Comput. Sci. Eng., 2: 1159-1164.

Murty, M.N. and V.S. Devi, 1988. Pattern recognition: An algorithmic approach. Springer, Universities Press.

Nicolás, F.G., C.I. Chesñevar and S.M. Castro, 2012. Automatic vehicle identification for Argentinean license plates using intelligent template matching. Patt. Recog. Lett., 33: 1066-1074.

DOI: $10.1016 /$ j.patrec.2012.02.004

Patrick, S.P.W., 2011. Pattern recognition, machine intelligence and biometrics. Higher Education Press, Beijing and Springer.

Rob, G.J.W. and H.N.W. Peter, 2011. Identity verification using computer vision for automatic garage door opening. IEEE Trans. Consumer Electron., 57: 906-914. DOI: 10.1109/TCE.2011.5955239

Sandeep, S., S.K. Das, N. Paul and S. Kundu, 2013. Optical character recognition using 40- point feature extraction and artificial neural network. Int. J. Adv. Res. Comput. Sci. Software Eng., 3: 495-502.

Sang, K.K., D.W. Kim and H.J. Kim, 1996. A recognition of vehicle license plate using a genetic algorithm based segmentation. Proceedings of the International Conference on Image Processing, Sep. 19-19, IEEE Xplore Press, South Korea, pp: 661-664. DOI: $10.1109 /$ icip.1996.560964

Sedighi, A. and M. Vafadust, 2011. A new and robust method for character segmentation and recognition in license plate images. Expert Syst. Applic., 38: 13497-13504. DOI: 10.1016/j.eswa.2011.02.030

Singh, D., J.P. Saini and D.S. Chauhan, 2014. Analysis of handwritten Hindi character recognition using advanced feature extraction technique and back propagation neural network. Int. J. Comput. Applic.
Sunghoon, K., D. Kim, Y. Ryu and G. Kim, 2002. A robust license-plate extraction method under complex image conditions. Proceedings of the 16th International Conference on Pattern Recognition, Aug. 11-15, IEEE Xplore press, South Korea, pp: 216-219. DOI: 10.1109/ICPR.2002.1047833

Syed, F.A., M. Moinuddin, W. Taj, F. Jaffri and S. Tasneem, 2013. A neural network based Pakistani license plate recognition system and controlling mechanism. Asian J. Eng. Sci. Technol., 3: 69-73.

Tahir, A., H.A. Habib and M.F. Khan, 2010. License plate recognition algorithm for Pakistani license plates. Canad. J. Image Process. Comput. Vis., 1: 30-36.

Vahid, A. and A. Alireza, 2009. An edge-based coloraided method for license plate detection. Image Vis. Comput., 27: 1134-1142. DOI: 10.1016/j.imavis.2008.10.012

Vladimir, S.V., G. Gluhchev and D. Dimov, 2006. Towards a multinational car license plate recognition system. Mach. Vis. Applic., 17: 173-183.

DOI: $10.1007 / \mathrm{s} 00138-006-0023-5$

Wenjing, J., H. Zhang and X. He, 2007. Region-based license plate detection. Comput. Applic., 30: 1324-1333. DOI: 10.1016/j.jnca.2006.09.010

Yo-Ping, H., C.H. Chen, Y.T. Chang and F.E. Sandnes, 2009. An intelligent strategy for checking the annual inspection status of motorcycles based on license plate recognition. Expert Syst. Applic., 36: 9260-9267. DOI: 10.1016/j.eswa.2008.12.006

Yusuf, P., N. Akhtar and F. Parwej, 2014. The Kingdom of Saudi Arabia vehicle license plate recognition using learning vector quantization artificial neural network. Int. J. Comput. Applic.

Zounemat-Kermani, M., A. Beheshti, B. Ataie-Ashtiani and S. Sabbagh-Yazdi, 2009. Estimation of currentinduced scour depth around pile groups using neural network and adaptive neuro-fuzzy inference system. Applied Soft Comput., 9: 746-755. DOI: 10.1016/j.asoc.2008.09.006 